\title{
RẼGLEMENTS MINIERS
}

1390-1512 
ECOLE PRATIQUE DES HAUTES ETUDES

VIE SECTION

\title{
DOCUMENTS ET RECHERCHES
}

\author{
SUR L'ÉCONOMIE DES PAYS \\ BYZANTINS, ISLAMIQUES ET SLAVES \\ ET LEURS RELATIONS COMMERCIALES \\ AU MOYEN AGE
}

SOUS LA DIRECTION DE

PAUL LEMERLE

VII

PARIS

MOUTON \& CO

1964

LA HAYE 
ECOLE PRATIQUE DES HAUTES ÉTUDES
VIe SECTION

\author{
LES ACTES DES PREMIERS SULTANS \\ CONSERVÉS DANS LES MANUSCRITS TURCS \\ DE LA BIBLIOTHẼQUE NATIONALE A PARIS
}

II

\title{
RẼGLEMENTS MINIERS \\ 1390-1512
}

PAR

NICOARĂ BELDICEANU 


\begin{abstract}
CET OUVRAGE A É TÉ PUBLIÉ AVEC LE CONCOURS DU CENTRE NATIONAL DE LA RECHERCHE SCIENTIFIQUE
\end{abstract}

(C) 1964 by Mouton \& Co and École pratique des Hautes Etudes

No part of this book may be translated or reproduced in any form by print, photoprint, or microfilm without written permission from the publishers.

Printed in France 
Piae memoriae magistri et amici carissimi V. Papacostea 
